\title{
Hydroxychloroquine and risk of development of cancers: a nationwide population-based cohort study
}

This article was published in the following Dove Press journal:

Therapeutics and Clinical Risk Management

\author{
I-Chieh Mao,' Ching-Yeh \\ Lin, ${ }^{2}$ Chia-Lin Wu, ${ }^{3,4}$ Chew- \\ Teng Kor, ${ }^{5,6}$ Chia-Chu \\ Chang ${ }^{3,7-9}$
}

'Division of Critical Care, Department of Internal Medicine, Changhua Christian Hospital, Changhua, Taiwan; ${ }^{2}$ Division of Hematology and Oncology, Department of Internal Medicine, Changhua Christian Hospital, Changhua, Taiwan; ${ }^{3}$ Division of Nephrology, Department of Internal Medicine, Changhua Christian Hospital, Changhua, Taiwan; ${ }^{4}$ Institute of Clinical Medicine, National YangMing University, Taipei, Taiwan; ${ }^{5}$ Internal Medicine Research Center, Changhua Christian Hospital, Changhua, Taiwan; ${ }^{6} \mathrm{Graduate}$ Institute of Statistics and Information Science, National Changhua University of Education, Changhua, Taiwan; ${ }^{7}$ Department of Internal Medicine, Kuang Tien General Hospital, Taichung, Taiwan; ${ }^{8}$ School of Medicine, Chung-Shan Medical University, Taichung, Taiwan; ${ }^{9}$ Department of Nutrition, Hungkuang University, Taichung, Taiwan

Correspondence: Chia-Chu Chang Division of Nephrology, Department of Internal Medicine, Changhua Christian Hospital, I 35 Nanhsiao St, Changhua 500, Taiwan

Tel +88647238595 ext I03।

Fax +886 47228289

Email chiachu@cch.org.tw

Chew-Teng Kor

Internal Medicine Research Center,

Changhua Christian Hospital, I 35

Nanhsiao Street, Changhua 500, Taiwan

Tel +88647238595 ext 4738

Fax +88622826 4049

Email 179297@cch.org.tw
Background: Hydroxychloroquine (HCQ), one of the disease-modifying antirheumatic drugs, may lead to an inhibition of autophagy. Autophagy, an intracellular self-defense mechanism for the lysosomal degradation of cytoplasmic components such as damaged organelles, plays a role in protecting against neoplasm growth but is also vital for cancer cells due to an increased intracellular metabolic waste.

Methods: Taiwan National Health Insurance Database was subjected to analysis to investigate the effect of HCQ exposure on cancer risk in patients with autoimmune diseases. Cancer incidence between patients with or without at least 12-month HCQ use was compared by propensity score-matched landmark analysis. A total of 100,000 participants were enrolled, including 7,662 patients who were diagnosed with autoimmune diseases between January 1, 2000, and December 31, 2012.

Results: After propensity score matching, HCQ user and nonuser groups consist of 1,933 patients with a mean follow-up time of 7.82 and 6.7 years, respectively. During the follow-up period, 93 HCQ users and 77 HCQ nonusers developed cancers. Meanwhile, Kaplan-Meier estimates showed no difference in the overall incidence of cancer between HCQ users and nonusers.

Conclusion: This propensity score-matched study of Taiwanese patients with autoimmune diseases suggested that HCQ exposure did not increase the cancer risk.

Keywords: hydroxychloroquine, autophagy, cancer, autoimmune diseases, propensity score

\section{Introduction}

Hydroxychloroquine (HCQ) is a 4-aminoquinoline agent that has been used for $>50$ years to prevent or to treat malarial infections and later also to treat autoimmune diseases such as systemic lupus erythematosus and rheumatoid arthritis. ${ }^{1}$ Recently, HCQ has been demonstrated to have anticancer effects by inhibiting autophagy pathway in some cancer types, such as breast cancer, ${ }^{2}$ glioblastoma, lung cancer, multiple myeloma, pancreatic cancer, melanoma, hepatocellular carcinoma, and bladder cancer. ${ }^{1,3-5}$

Autophagy is an evolutionarily conserved, intracellular self-defense mechanism for the lysosomal degradation of cytoplasmic components. ${ }^{6}$ Damaged organelles and protein aggregates are sequestered into autophagic vesicles (also known as autophagosomes) that are subsequently degraded through fusion with lysosomes, which makes autophagy critical for the cellular remodeling ${ }^{7}$ and maintenance of intracellular homeostasis. ${ }^{8}$ In some stress conditions, such as infection, apoptosis, and cancer behaviors, autophagy is additionally upregulated to response difficult environmental disturbance. ${ }^{5}$ Therefore, autophagy plays an essential role in cell 
development, differentiation, normal growth, and immunity. In line with this notion, defected autophagy has been shown to involve in some clinical disorders, including infectious, ${ }^{9}$ neurodegenerative, ${ }^{10}$ and neoplastic ${ }^{11}$ diseases.

Interestingly, the effect of autophagy is a double-edged sword ${ }^{12}$ for cancer cells. As a tumor suppressor, autophagy prevents the accumulation of damaged proteins and organelles. ${ }^{6}$ As a tumor promotor, autophagy facilitates tumor growth and aggressiveness by surviving microenvironmental stress. ${ }^{6}$ Cancer cells rely and are even more dependent on autophagy due to increased metabolic and biosynthetic demands imposed by deregulated proliferation. ${ }^{13}$

No doubt, autoimmune diseases, representing chronic inflammation status, have a clear association with cancer. ${ }^{14}$ Whether administration of HCQ, which leads to the inhibition of autophagy in patients with autoimmune diseases, increases the risk of cancer development is not clearly described. It is important to eliminate this doubt to ensure the safety of HCQ use in such high-risk population. Our study aimed to clarify whether HCQ use is associated with increased risk of cancers. In this retrospective study involving a large-scale nationwide cohort, we evaluated the effect of HCQ exposure on the development of cancers in patients with autoimmune diseases.

\section{Methods}

\section{Data source}

Data were retrieved from the Taiwan's National Health Insurance Research Database (NHIRD), which includes all claims data from the National Health Insurance program. ${ }^{15}$ These claims include demographic data, ambulatory care, record of clinic visits, hospital admissions, dental services, prescriptions, and disease status. The National Health Insurance program, which was started in Taiwan in March 1995, covers $>99 \%$ of the total population or $\sim 23$ million people. Researchers can apply for specific dataset such as cancer or catastrophic illness dataset and longitudinal dataset containing a random sample of 1 million NHI enrollees. Diagnostic codes for identifying diseases were based on ICD, Ninth Revision, Clinical Modification (ICD-9-CM). The drug prescriptions were managed according to Anatomical Therapeutic Chemical (ATC) codes defined by World Health Organization (WHO). Defined daily dose (DDD) was used to measure the medication consumption, and it is $516 \mathrm{mg}$ for HCQ defined by WHO. Because anonymized and encrypted secondary data were analyzed, informed consent was exempt in this study. Ethics approval was obtained from the Institutional Review Board of the Changhua Christian Hospital (approval number 180604).

\section{Study population}

Patients with autoimmune diseases were identified by using ICD-9-CM code 710.2 for Sjögren's syndrome, 696.0-696.1 for psoriasis, 714.0 for rheumatoid arthritis, 700 for systemic lupus erythematosus, 710.1 for scleroderma, and 710.4 for polymyositis. Cancer events were identified from the Registry of Catastrophic Illness Patient Database, which is a subset of the NHIRD, by excluding patients with the history of cancer before the index date, aged $<18$ years, and survived or being followed for $<1$ year. If the patients are diagnosed with a new cancer within 1 year, we assumed that the cancer may precede than the autoimmune diseases and may not be related to the use of HCQ. Exposure to HCQ (HCQ user) was defined as a pharmacological treatment of HCQ given within 12 months after the diagnosis of systemic autoimmune diseases. The index date on which the 12 months after diagnosis was defined as the index date to ensure that each patient had enough observation window for HCQ exposure. In addition, the index date was set-up at 366 days following the diagnosis of autoimmune diseases to avoid immortal time bias. The aim of this propensity score-matched study is to investigate the effect of HCQ on cancer incidence. Propensity score was calculated by logistic regression models to indicate the conditional probability of receiving HCQ and then adjusted by age, gender, autoimmune diseases, socioeconomic factors, medications, and comorbidities. Eventually, HCQ-exposed patients and nonexposed patients were matched at a ratio of $1-1$.

\section{Outcome measures and relevant variables}

The catastrophic illness registry was used to identify cancer cases (ICD-9-CM codes 140-208). Major comorbid diseases diagnosed before the index date were defined as baseline comorbidities based on claims data. These comorbidities included hypertension, diabetes mellitus (DM), hyperlipidemia, coronary artery disease (CAD), congestive heart failure (CHF), stroke, chronic obstructive pulmonary disease (COPD), and alcohol-related diseases (alcoholism, alcoholic liver disease, and alcoholic gastritis). Charlson's comorbidity index score was used to quantify baseline comorbidities. ${ }^{16}$

\section{Statistical analysis}

Demographic and clinical characteristics in the HCQ user and HCQ nonuser cohorts were summarized using proportions and mean $\pm \mathrm{SD}$. Chi square tests and Student's $t$-tests were used to compare the distributions of discrete and continuous variables, respectively. Cox's proportional hazard models were used to estimate the relative risk of developing cancers in the HCQ user cohort compared with that in the HCQ 
nonuser cohort. Confounders, including age, gender, type of autoimmune diseases, and propensity score, were adjusted in multivariate Cox's analysis with competing risks (Fine-Gray subdistribution hazards models) of death to estimate adjusted hazard ratios (aHRs). To determine the dose-response relation, we estimated the risk of cancer according to the cumulative DDD (cDDD) during the 1-year exposure period (DDD 1-142 or $>142 \mathrm{mg}$ ) and the prescribed daily dose ( $\leq 200,201-400$, or $>400 \mathrm{mg}$ ) compared with HCQ nonuser. Cumulative incidence of cancers was calculated using the Kaplan-Meier estimation and compared using Log-rank tests. To assess the reliability of our results, five sensitivity analyses were performed to ascertain our results. First of all, clinical variables (demographics, comorbidities, and long-term medications) were adjusted in multivariable Cox proportional hazard model. Second, we evaluated misclassification bias by defining HCQ use at intervals 90, 150, and 180 days after the initial diagnosis of autoimmune diseases. Third, an as-treat model for patients who discontinued HCQ use was censored. Fourth, we evaluated the patients who were followed up for $>7$ and 10 years due to the evolutionary time to tumor. Fifth, we removed patients with other immunosuppressants in order to minimize potential effects on unbalanced covariate after propensity score matched. All statistical analyses were performed using the SAS 9.4 software (SAS Institute Inc., Cary, NC, USA). Two-tailed $P$-values $<0.05$ were considered statistically significant.

\section{Results}

Through the subject selection process shown in Figure 1, a total of 100,000 participants were enrolled to include 7,662 patients diagnosed with autoimmune diseases between January 1, 2000, and December 31, 2013. During this process, 1,112 patients were excluded and 6,541 patients were eligible for subsequent analysis, including 3,408 HCQ users and 3,133 HCQ nonusers. After propensity score matching, 1,993 subjects were assigned to each group. Variables included in the propensity score calculation did not significantly differ between HCQ user and nonuser after matching, which confirms the success of matching (Table 1).

Table 1 shows the baseline characteristics of study population to reveal a similar age distribution in both cohorts,

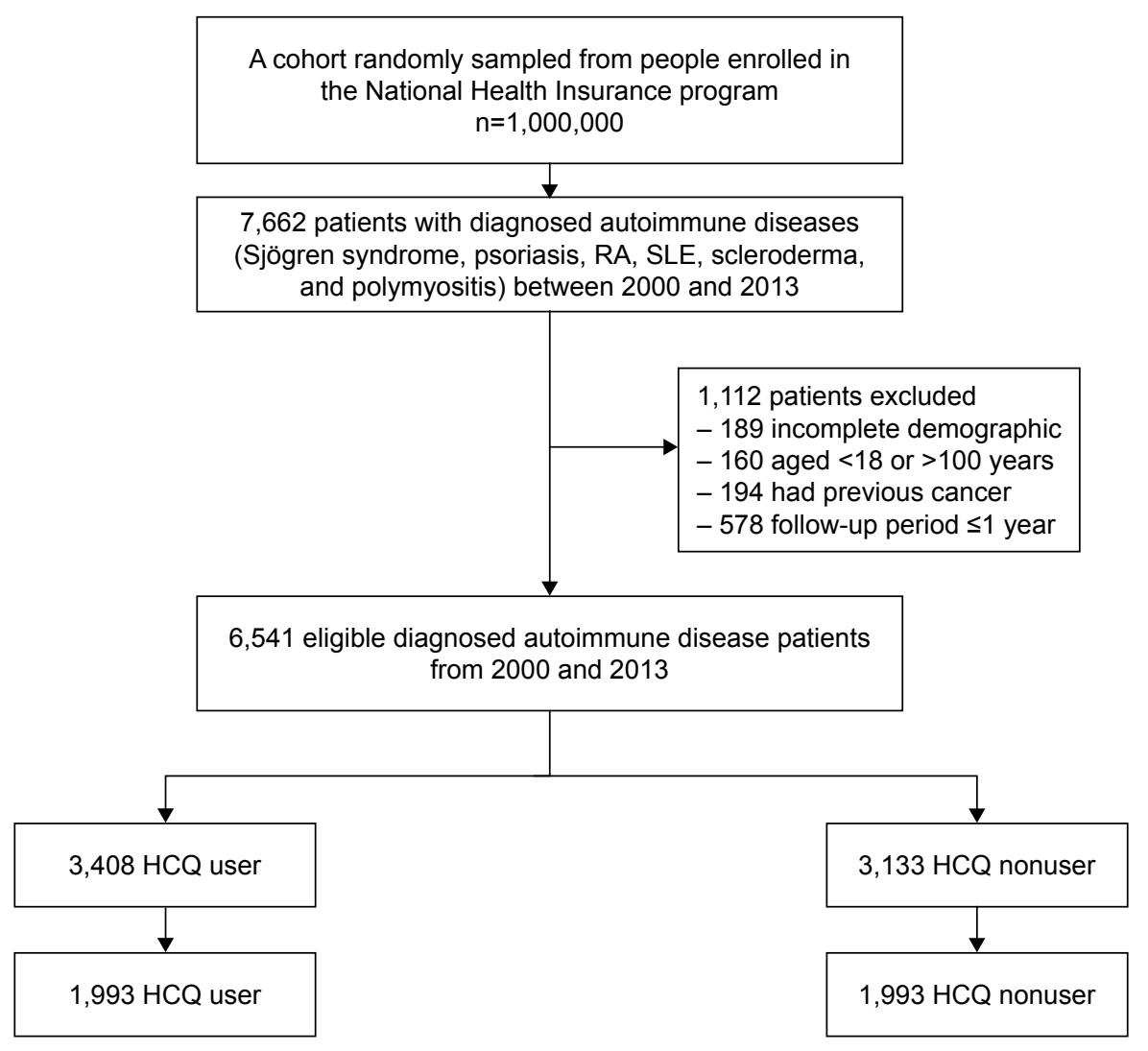

Figure I Flow chart for subject selection process.

Notes: From 2000 to 2013, 7,662 patients diagnosed with RA, SLE, psoriasis, Sjögren's syndrome, scleroderma, or polymyositis were identified in the NHIRD. A total of $6,54 \mathrm{I}$ patients were eligible for subsequent analysis after exclusion. After propensity score matching, I,993 subjects were assigned to each group. A total of I,II2 patients were excluded: I) 189 patients for incomplete demographics, 2) 160 patients for being an age of $<18$ or $>100$ years, 3) 194 patients for having a previous cancer, and 4) 578 patients for having a follow-up period of $\leq$ I year.

Abbreviations: HCQ, hydroxychloroquine; NHIRD, National Health Insurance Research Database. 
Table I Demographics and clinical characteristics of the study population

\begin{tabular}{|c|c|c|c|c|c|c|}
\hline \multirow[t]{2}{*}{ Characteristic } & \multicolumn{3}{|c|}{ Before propensity score-matched data } & \multicolumn{3}{|c|}{ After propensity score-matched data } \\
\hline & $\begin{array}{l}\mathrm{HCQ}=0 \\
(\mathbf{N}=3,133)\end{array}$ & $\begin{array}{l}\mathrm{HCQ}=\mathrm{I} \\
(\mathrm{N}=\mathbf{3}, \mathbf{4 0 8})\end{array}$ & $P$-value & $\begin{array}{l}\mathrm{HCQ}=0 \\
(\mathrm{~N}=1,993)\end{array}$ & $\begin{array}{l}\mathrm{HCQ}=\mathrm{I} \\
(\mathbf{N}=\mathbf{I}, 993)\end{array}$ & $P$-value \\
\hline Age (years) & $49.77 \pm 14.96$ & $50.16 \pm 15.34$ & 0.293 & $50.95 \pm 13.66$ & $50.96 \pm 13.69$ & 0.983 \\
\hline Gender, male, n (\%) & $900(28.73)$ & $604(17.72)$ & $<0.001$ & $312(15.65)$ & $312(15.65)$ & 1.000 \\
\hline \multicolumn{7}{|l|}{ Autoimmune diseases, $\mathrm{n}(\%)$} \\
\hline Rheumatoid arthritis & $\mathrm{I}, 370(43.73)$ & $\mathrm{I}, 565(45.92)$ & & $\mathrm{I}, 098$ (55.09) & $\mathrm{I}, 098(55.09)$ & 1.000 \\
\hline Systemic lupus erythematosus & $242(7.72)$ & $612(17.96)$ & & $159(7.98)$ & $159(7.98)$ & \\
\hline Sjögren's syndrome & $1,008(32.17)$ & I,I62 (34.I) & & $720(36.13)$ & $720(36.13)$ & \\
\hline Psoriasis & $428(13.66)$ & $15(0.44)$ & & $7(0.35)$ & $7(0.35)$ & \\
\hline Scleroderma & $5 \mathrm{I}(1.63)$ & $36(1.06)$ & & $8(0.4)$ & $8(0.4)$ & \\
\hline Polymyositis & $34(1.09)$ & $18(0.53)$ & & I (0.05) & I (0.05) & \\
\hline \multicolumn{7}{|l|}{ Geographic location, n (\%) } \\
\hline Northern Taiwan & I,494 (47.69) & I,463 (42.93) & $<0.001$ & $93 I(46.7 I)$ & $919(46.11)$ & 0.796 \\
\hline Central Taiwan & $856(27.32)$ & 97I (28.49) & & $543(27.25)$ & $558(28)$ & \\
\hline Southern Taiwan & $723(23.08)$ & 911 (26.73) & & $470(23.58)$ & $475(23.83)$ & \\
\hline Eastern Taiwan and Islands & $60(1.92)$ & $63(1.85)$ & & $49(2.46)$ & $4 \mathrm{I}(2.06)$ & \\
\hline Clinic visit frequency & $28.82 \pm 18.69$ & $31.92 \pm 17.29$ & $<0.001$ & & & \\
\hline Monthly income, NTD & $|8,972.97 \pm| 6,4|| .45$ & $|7,407.22 \pm| 4,733.16$ & $<0.001$ & $17,580.58 \pm \mid 4,579.27$ & $|8,30| .88 \pm|5| 24.6 \mid$, & 0.125 \\
\hline \multicolumn{7}{|l|}{ Comorbidities } \\
\hline CCIS & $1.33 \pm 1.49$ & $1.34 \pm 1.53$ & $0.74 I$ & $1.35 \pm 1.47$ & $1.32 \pm 1.5$ & 0.639 \\
\hline Hypertension, n (\%) & $716(22.85)$ & $802(23.53)$ & 0.516 & $453(22.73)$ & $453(22.73)$ & 1.000 \\
\hline Hyperlipidemia, n (\%) & 449 (14.33) & $444(13.03)$ & 0.125 & $284(14.25)$ & $276(13.85)$ & 0.715 \\
\hline Diabetes mellitus, n (\%) & $273(8.7 I)$ & $274(8.04)$ & 0.325 & $164(8.23)$ & $175(8.78)$ & 0.532 \\
\hline CAD, n (\%) & $28 \mathrm{I}(8.97)$ & $293(8.6)$ & 0.596 & I6I (8.08) & $165(8.28)$ & 0.817 \\
\hline CHF, n (\%) & $76(2.43)$ & $96(2.82)$ & 0.323 & $45(2.26)$ & $48(2.4 I)$ & 0.753 \\
\hline Stroke, n (\%) & $156(4.98)$ & $172(5.05)$ & 0.900 & $92(4.62)$ & $90(4.52)$ & 0.879 \\
\hline COPD, n (\%) & $364(11.62)$ & $420(12.32)$ & 0.380 & 229 (II.49) & $217(10.89)$ & 0.547 \\
\hline Alcohol-related disease, n (\%) & $21(0.67)$ & $21(0.62)$ & 0.784 & $10(0.5)$ & II $(0.55)$ & 0.827 \\
\hline \multicolumn{7}{|l|}{ Long-term medications, n (\%) } \\
\hline Antidiabetic drugs & $236(7.53)$ & $247(7.25)$ & 0.660 & $130(6.52)$ & $142(7.12)$ & 0.451 \\
\hline Antihypertensive drugs & $923(29.46)$ & I,I2I (32.89) & 0.003 & $557(27.95)$ & $544(27.3)$ & 0.645 \\
\hline ACEls/ARBs & $475(15.16)$ & $573(16.8 I)$ & 0.069 & $26 I(13.1)$ & $276(13.85)$ & 0.487 \\
\hline Diuretics & $28 I(8.97)$ & $372(10.92)$ & 0.009 & $162(8.13)$ & $160(8.03)$ & 0.907 \\
\hline NSAIDs & $465(14.84)$ & $627(18.4)$ & $<0.001$ & $291(14.6)$ & $297(14.9)$ & 0.789 \\
\hline Analgesic drugs other than & $497(15.86)$ & $691(20.28)$ & $<0.001$ & $285(14.3)$ & $300(15.05)$ & 0.502 \\
\hline \multicolumn{7}{|l|}{ NSAIDs } \\
\hline Glucocorticoids & $477(15.23)$ & $\mathrm{I}, \mathrm{I05}(32.42)$ & $<0.001$ & $262(13.15)$ & $273(13.7)$ & 0.609 \\
\hline TNF- $\alpha$ inhibitors & $117(3.73)$ & $196(5.75)$ & $<0.001$ & $63(3.16)$ & $82(4.11)$ & 0.108 \\
\hline Other immunosuppressants & $362(11.55)$ & $523($ (I5.35) & $<0.001$ & $108(5.42)$ & $164(8.23)$ & $<0.001$ \\
\hline Propensity score & $0.49 \pm 0.1$ & $0.55 \pm 0.12$ & $<0.001$ & $0.5 \pm 0.09$ & $0.5 \pm 0.09$ & 0.874 \\
\hline cDDD of $\mathrm{HCQ}$ within I year & $0 \pm 0$ & $111.13 \pm 86.75$ & $<0.001$ & $0 \pm 0$ & $99.83 \pm 83.73$ & $<0.001$ \\
\hline \multicolumn{7}{|l|}{ Outcome, $n(\%)$} \\
\hline Cancer & I35 (4.3 I) & $123(3.6 I)$ & & $93(4.67)$ & $77(3.86)$ & \\
\hline Death & $184(5.87)$ & $200(5.87)$ & & III (5.57) & $113(5.67)$ & \\
\hline Follow-up time (years) & $7.64 \pm 3.7$ & $6.75 \pm 3.73$ & $<0.001$ & $7.82 \pm 3.68$ & $6.7 \pm 3.73$ & $<0.001$ \\
\hline
\end{tabular}

Abbreviations: CAD, coronary artery disease; cDDD, cumulative defined daily dose; CHF, congestive heart failure; HCQ, hydroxychloroquine.

with a mean age of $50.95 \pm 13.66$ and $50.96 \pm 13.69$ years in HCQ user and nonuser groups, respectively. With female (84.35\%) accounting for the majority, all patients were diagnosed with autoimmune diseases, including rheumatoid arthritis (55.09\%), Sjögren's syndrome (36.13\%), systemic lupus erythematosus $(7.98 \%)$, scleroderma $(0.4 \%)$, psoriasis $(0.35 \%)$, and polymyositis $(0.05 \%)$. Most of the population were from northern Taiwan without significant difference regarding monthly income. The comorbidities, including hypertension, hyperlipidemia, DM, COPD, and alcoholrelated diseases, are similar between HCQ user and HCQ nonuser groups. However, HCQ users still have a significantly higher rate of taking other immunosuppressants, such as methotrexate, leflunomide, sulfasalazine, and azathioprine. 


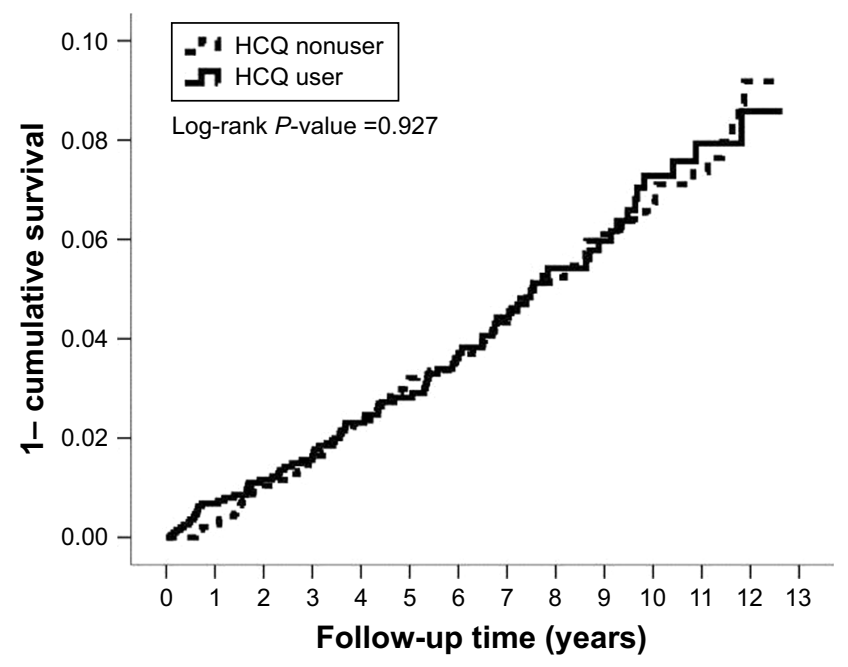

Figure 2 Kaplan-Meier curves for cumulative incidence of cancer, HCQ nonuser and user.

Note: No significant different cumulative incidence of cancer between HCQ user and nonuser.

Abbreviation: $\mathrm{HCQ}$, hydroxychloroquine.

The mean follow-up duration is 7.82 and 6.7 years, respectively, in HCQ nonuser and user groups.

Results in Figures 2-4 revealed the relationship between cancer risk and HCQ and dose-response of HCQ. KaplanMeier curve showed no significant different cumulative incidence of cancer between HCQ user and nonuser ( $\log$ rank test $P$-value $=0.927)($ Figure 2$)$. The incidence of cancer was not significantly increased in the larger cumulative daily dose of HCQ group (Figure 3, $P=0.958$ ). In Figure 4, our results suggested that prescribed daily dose did not affect the incidence of cancer significantly. In extended

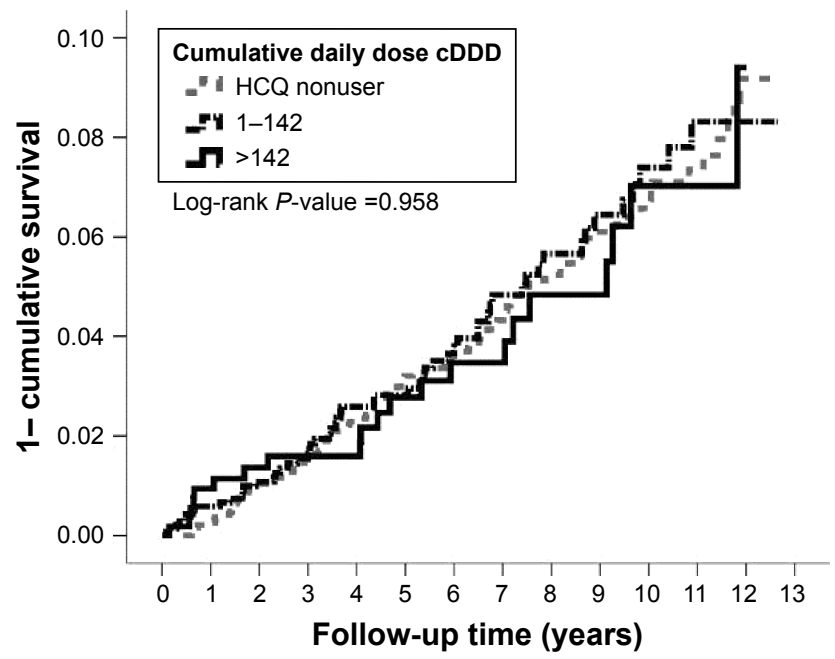

Figure 3 Kaplan-Meier curves for cumulative incidence of cancer with various CDDDs of HCQ.

Note: The incidence of cancer was not significantly increased in larger cDDD of $\mathrm{HCQ}$ group.

Abbreviations: cDDDs, cumulative defined daily doses; $\mathrm{HCQ}$, hydroxychloroquine.

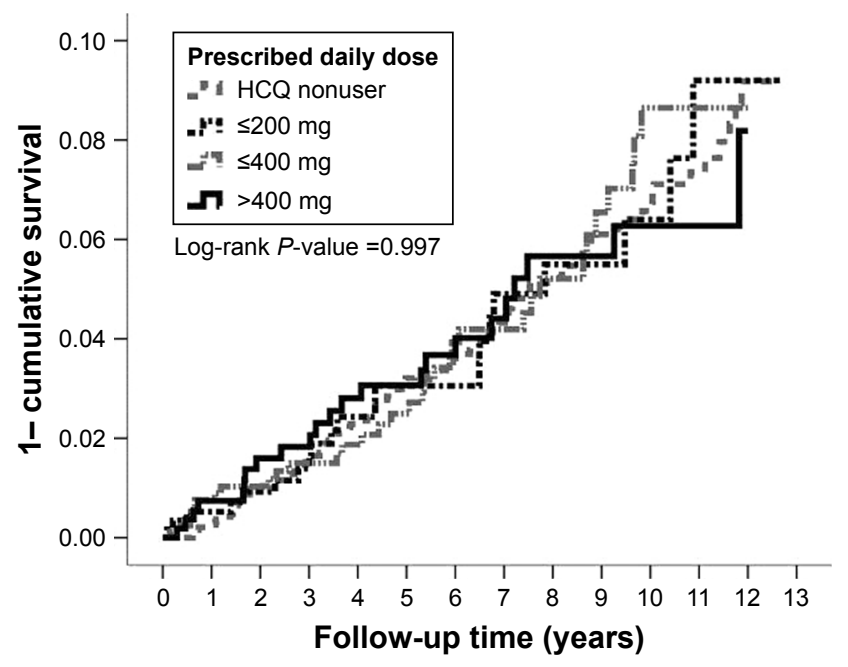

Figure 4 Kaplan-Meier curves for cumulative incidence of cancer with various prescribed daily dose of $\mathrm{HCQ}$.

Note: Prescribed daily dose did not affect the incidence of cancer significantly. Abbreviation: $\mathrm{HCQ}$, hydroxychloroquine.

Cox proportional hazards models (Table 2), confounding factors, including age, gender, type of autoimmune diseases, and propensity score, were adjusted and the aHRs of cancer were 1.027 (95\% CI: 0.76-1.39) in the HCQ user group, 1.088 (95\% CI: $0.68-1.75)$ in the group with prescribed daily dose $\leq 200 \mathrm{mg}, 1.051$ (95\% CI: 0.71-1.57) in the group with prescribed daily dose $201-400 \mathrm{mg}$, and 0.986 (95\% CI: $0.63-1.55)$ in the group with prescribed daily dose $>400 \mathrm{mg}$. For cDDDs, the hazard ratio was 1.077 (95\% CI: 0.77-1.50) in 1-142 cDDDs' group and 0.933 (95\% CI: $0.58-1.50)$ in $>142$ cDDDs' group. Therefore, HCQ did not showed significant increase in cancer risk. Similar to that from primary analyses, results from the subgroup analysis (Table 3) demonstrated that there was no significant difference in the risk of cancer between HCQ user and nonuser across different ages, genders, comorbidities, and autoimmune diseases. Moreover, none of these subgroups significantly interacted with HCQ treatment (all interactions $P>0.05$ ). As shown in Table 4, there was no difference in risk for specific cancers between two cohorts, in both unadjusted and adjusted models.

Regarding the reliability of our main results, results of five steps of sensitivity analyses shown in Table 5 have showed consistence with those of our primary analyses.

\section{Discussion}

This is the first population-based study to investigate the effects of HCQ on the incidence of malignancy in patients with autoimmune diseases. Our evidence suggests that HCQ use is not associated with an increased risk of cancers in 


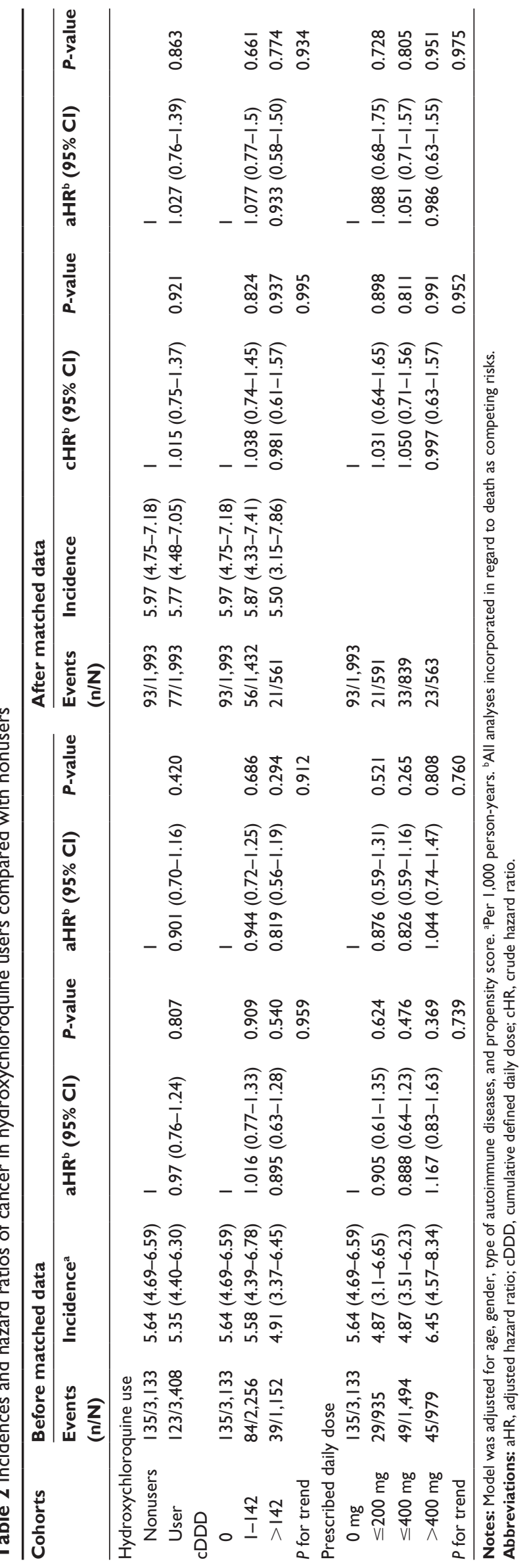

patients with autoimmune diseases. After adjustment for cancer risk factors and covariates including age, gender, and autoimmune types, HCQ still does not increase the risk in patients with autoimmune diseases both in hematological and solid malignancies (Table 4).

Recently, the safety issue of long-term HCQ therapy mainly focuses on retinopathy. ${ }^{17}$ To our knowledge, little attention has been paid to the safety concern regarding the effect on cancer development of HCQ. On the contrary, growing data and researches are emerging on the anticancer effects of HCQ and HCQ is mostly often administered in combination with other anticancer agents. Multiple hypotheses have been proposed on how HCQ exerts their anticancer activities. The most popular hypothesis is that the antineoplastic activity of HCQ probably stems from the direct inhibition of autophagy pathway ${ }^{18}$ to augment the efficacy of anticancer agents. ${ }^{19}$ As a tumor suppressor, HCQ inhibits autophagy to suppress the growth of established tumors, which had been illuminated in cell and mice studies. ${ }^{20-22}$ In several preclinical studies, administration of HCQ can disable autophagy pathway through the inhibition of fusion of autophagosomes with lysosomes and their degradation. ${ }^{23}$ Up to date, there are $>20$ ongoing trials involving HCQ on human cancer treatment on ClinicalTrials.gov.

Our study used a real-world large nationwide populationbased cohort to understand whether HCQ has any effect on the incidence of cancers. The results did not support that HCQ use has any effect on cancer risk, regardless of the cDDDs or prescribed daily doses. Therefore, HCQ can safely be used as a disease-modifying antirheumatic drug for autoimmune diseases without concerns of its autophagy inhibition ability that would potentially promote the risk of cancer development. It is worth noting that in our subgroup analysis, there is a trend of decreasing incidence of cancer in elderly patients after adjusting confounding factors. Therefore, it may need more investigation to clarify if HCQ has a protective benefit of cancer development in elderly patient with autoimmune diseases.

Some possible explanations may be taken into consideration for the interpretation of our observations. First of all, patients with autoimmune diseases are already at a higher risk of cancers than general population. ${ }^{24}$ Unregulated inflammation chronically provokes cellular malignant transformation and carcinogenesis in surrounding tissues. Compared to this strong trigger factor, the contribution of the carcinogenicity of HCQ may be neglected. Second, the usual dosage of HCQ used to treat autoimmune disease patients is often $<400 \mathrm{mg}$ daily while the dosage of HCQ to be antineoplastic or able to inhibit autophagy is required as high as up to $1,000 \mathrm{mg} .{ }^{25}$ HCQ at a lower dosage may only exert limited ability for 


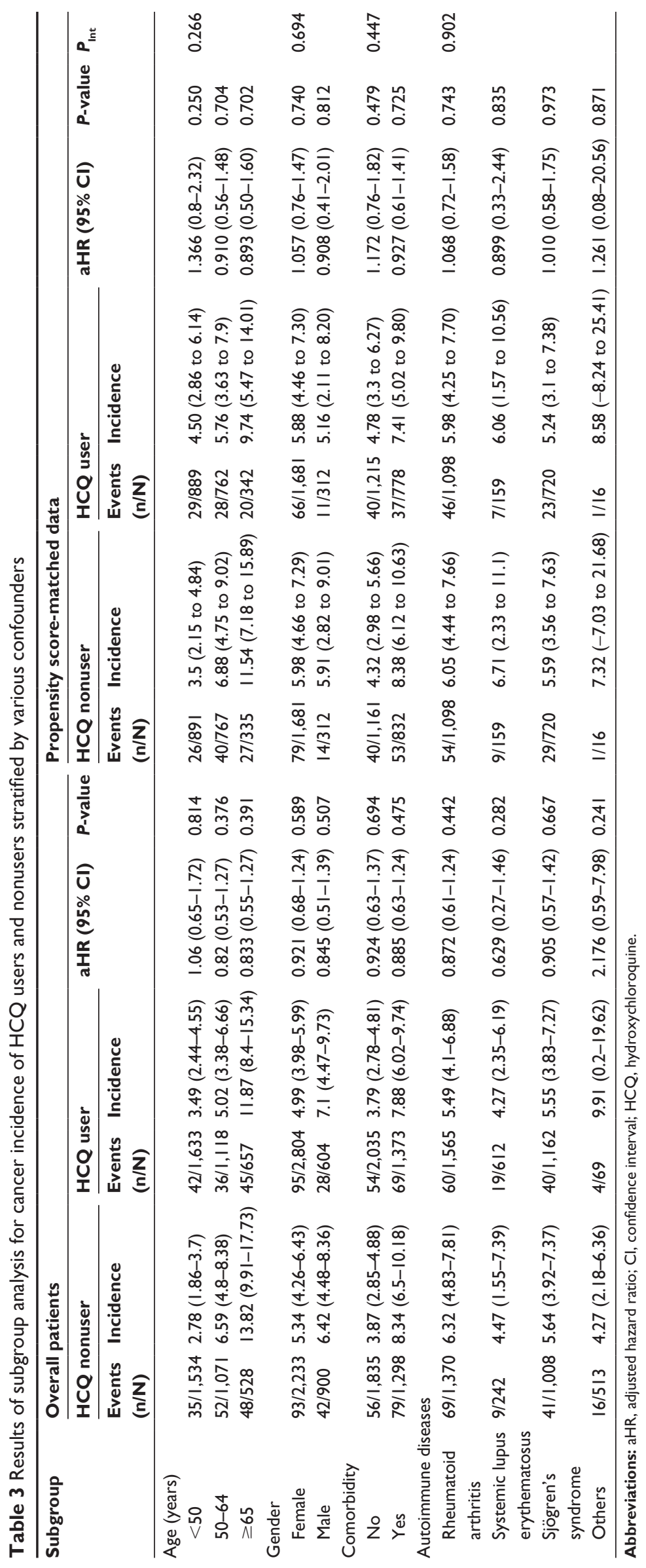


Table 4 Risk of solid cancer and hematological cancer

\begin{tabular}{|c|c|c|c|c|c|c|}
\hline Cancer & $\begin{array}{l}\text { Event in patients } \\
\text { without } \mathrm{HCQ} \text { user }\end{array}$ & $\begin{array}{l}\text { Event in patients } \\
\text { with } \mathrm{HCQ} \text { user }\end{array}$ & cHR $(95 \% \mathrm{CI})$ & $P$-value & aHR $(95 \% \mathrm{Cl})$ & $P$-value \\
\hline Hematological malignancy & 1 & 1 & $1.07(0.07-17.17)$ & 0.962 & $0.953(0.05-16.52)$ & 0.9737 \\
\hline Solid cancer & 134 & 122 & $0.969(0.76-1.24)$ & 0.8029 & $0.9(0.7-1.16)$ & 0.4172 \\
\hline Head and neck & 6 & 10 & $1.497(0.53-4.2)$ & 0.4431 & $1.718(0.57-5.18)$ & 0.3363 \\
\hline Esophagus & 2 & 0 & - & & - & \\
\hline Stomach & 4 & 10 & $2.442(0.78-7.67)$ & 0.1261 & I.858 (0.58-5.98) & 0.2984 \\
\hline Small intestine & 0 & I & - & & - & \\
\hline Colon & 16 & 16 & $1.076(0.54-2.15)$ & 0.8361 & $0.925(0.45-1.89)$ & 0.8299 \\
\hline Liver & 16 & 16 & $1.059(0.53-2.12)$ & 0.8711 & $1.167(0.57-2.4)$ & 0.6752 \\
\hline Pancreas & 2 & 0 & - & & - & \\
\hline Lung & 14 & 15 & $1.128(0.54-2.34)$ & 0.7457 & $1.019(0.48-2.16)$ & 0.9616 \\
\hline Skin & 0 & I & - & & - & \\
\hline Female breast & 31 & 26 & $0.791(0.47-1.33)$ & 0.3792 & $0.75(0.43-1.3)$ & 0.3025 \\
\hline Uterus & 10 & 10 & $0.95 I(0.4-2.29)$ & 0.9112 & $0.74 \mid(0.3-\mid .84)$ & 0.5189 \\
\hline Prostate & 7 & 2 & $0.55 \mathrm{I}(0.12-2.5)$ & 0.4395 & $0.438(0.09-2.1)$ & 0.3019 \\
\hline Bladder & 6 & 5 & $0.883(0.27-2.89)$ & 0.8369 & $0.94 \mid(0.28-3.2)$ & 0.9217 \\
\hline Kidney & 8 & 3 & $0.446(0.12-1.64)$ & 0.224 & $0.484(0.13-1.86)$ & 0.2915 \\
\hline Brain & I & 0 & - & & - & \\
\hline Thyroid & 4 & 5 & $1.313(0.35-4.89)$ & 0.6844 & $1.532(0.39-5.95)$ & 0.5377 \\
\hline
\end{tabular}

Abbreviations: aHR, adjusted hazard ratio; cHR, crude hazard ratio; $\mathrm{Cl}$, confidence interval; $\mathrm{HCQ}$, hydroxychloroquine.

inhibiting autophagy and eventually no apparent influence on cancer development.

The strength of this study was primarily based on the use of longitudinal population-based data, which represents the general population in Taiwan. However, this study has some potential limitations. First of all, the NHIRD does not include detailed information on socioeconomic status, smoking and betel nut chewing habits, dietary patterns, family history of

Table 5 Results of sensitivity analyses

\begin{tabular}{|c|c|c|c|c|}
\hline & \multicolumn{2}{|l|}{ Overall patients } & \multicolumn{2}{|c|}{ Propensity score-matched data } \\
\hline & aHR $(95 \% \mathrm{Cl})$ & $P$-value & aHR $(95 \% \mathrm{Cl})$ & $P$-value \\
\hline \multicolumn{5}{|c|}{ Multivariate model adjusted for covariate in Table I } \\
\hline Nonusers & 1 & & I & \\
\hline Users & $0.903(0.7-1.17)$ & 0.435 & $1.034(0.76-1.4)$ & 0.729 \\
\hline \multicolumn{5}{|c|}{ Hydroxychloroquine use at intervals 90 days after first } \\
\hline \multicolumn{5}{|c|}{ disease diagnosis } \\
\hline Nonusers & I & & I & \\
\hline Users & $0.929(0.72-1.2)$ & 0.569 & $0.946(0.69-1.3)$ & 0.729 \\
\hline \multicolumn{5}{|c|}{ Hydroxychloroquine use at intervals 150 days after first } \\
\hline \multicolumn{5}{|c|}{ disease diagnosis } \\
\hline Nonusers & 1 & & I & \\
\hline Users & $0.918(0.71-1.18)$ & 0.5119 & $0.962(0.7-|.3|)$ & 0.806 \\
\hline \multicolumn{5}{|c|}{ Hydroxychloroquine use at intervals 180 days after first } \\
\hline \multicolumn{5}{|c|}{ disease diagnosis } \\
\hline Nonusers & 1 & & 1 & \\
\hline Users & $0.940(0.73-1.21)$ & 0.6328 & $1.038(0.76-1.42)$ & 0.815 \\
\hline \multicolumn{5}{|c|}{ As treat model } \\
\hline Nonusers & 1 & & 1 & \\
\hline Users & $0.931(0.68-1.27)$ & 0.649 & $1.042(0.7-1.54)$ & 0.8387 \\
\hline \multicolumn{5}{|c|}{ Patients who were followed up for $>7$ years } \\
\hline Nonusers & 1 & & 1 & \\
\hline Users & $0.94(0.59-1.5)$ & 0.7956 & $1.024(0.62-1.69)$ & 0.9259 \\
\hline \multicolumn{5}{|c|}{ Patients who were followed up for $>10$ years } \\
\hline Nonusers & 1 & & 1 & \\
\hline Users & $1.109(0.48-2.59)$ & 0.8104 & I. $127(0.47-2.68)$ & 0.7861 \\
\hline \multicolumn{5}{|c|}{ After removal of patients with other immunosuppressants } \\
\hline Nonusers & 1 & & 1 & \\
\hline Users & $0.892(0.68-1.17)$ & 0.404 & $0.992(0.72-1.36)$ & 0.9581 \\
\hline
\end{tabular}

Abbreviations: aHR, adjusted hazard ratio; $\mathrm{Cl}$, confidence interval. 
cancers, and relevant biochemical parameters. Second, this study is not able to clearly elucidate the different effects of high $(\geq 1,000 \mathrm{mg})$ and low dosages of HCQ on the incidence of cancers. In such higher HCQ dose, whether there is any influence on cancer incidence in autoimmune diseases' patient remains to be investigated. Third, propensity was used to handle confounding by indication bias in our study. There may be residual confounders that have not been considered. Results derived from a retrospective cohort study are generally of lower statistical quality than those from prospective studies because of potential biases. Finally, as the majority of Taiwan's population is of Chinese ethnicity, the findings of this study may not be applicable to populations of other ethnic backgrounds.

\section{Conclusion}

This propensity score matching population-based retrospective cohort study revealed that Taiwanese patients with autoimmune diseases showed that HCQ had a neutral effect on cancer risk but a nonsignificant protective effect in elderly patients. HCQ is a widely and chronically used medication in autoimmune diseases and poses a potential effect of dysregulated tumor growth by inhibiting autophagy. However, the occurrence of malignancies should not be a concern according to our results.

\section{Disclosure}

The authors report no conflicts of interest in this work.

\section{References}

1. Verbaanderd C, Maes H, Schaaf MB, et al. Repurposing drugs in oncology $(\mathrm{ReDO})$ - chloroquine and hydroxychloroquine as anti-cancer agents. Ecancermedicalscience. 2017;11:1-35.

2. Cook KL, Wärri A, Soto-Pantoja DR, et al. Hydroxychloroquine inhibits autophagy to potentiate antiestrogen responsiveness in ER+ breast cancer. Clin Cancer Res. 2014;20(12):3222-3232.

3. Lin YC, Lin JF, Wen SI, et al. Chloroquine and hydroxychloroquine inhibit bladder cancer cell growth by targeting basal autophagy and enhancing apoptosis. Kaohsiung J Med Sci. 2017;33(5):215-223.

4. Manic G, Obrist F, Kroemer G, Vitale I, Galluzzi L. Chloroquine and hydroxychloroquine for cancer therapy. Mol Cell Oncol. 2014;1(1): e29911.
5. Amaravadi RK, Lippincott-Schwartz J, Yin XM, et al. Principles and current strategies for targeting autophagy for cancer treatment. Clin Cancer Res. 2011;17(4):654-666.

6. Yang ZJ, Chee CE, Huang S, Sinicrope FA. The role of autophagy in cancer: therapeutic implications. Mol Cancer Ther. 2011;10(9):1533-1541.

7. White $\mathrm{E}$. The role for autophagy in cancer. J Clin Invest. 2015;125(1): $42-46$.

8. Towers CG, Thorburn A. Therapeutic targeting of autophagy. EBioMedicine. 2016;14:15-23.

9. Deretic V, Saitoh T, Akira S. Autophagy in infection, inflammation and immunity. Nat Rev Immunol. 2017;13(10):722-737.

10. Nixon RA. The role of autophagy in neurodegenerative disease. Nat Med. 2013;19(8):983-997.

11. Mathew R, Karantza-Wadsworth V, White E. Role of autophagy in cancer. Nat Rev Cancer. 2007;7(12):961-967.

12. White E, Dipaola RS. The double-edged sword of autophagy modulation in cancer. Clin Cancer Res. 2009;15(17):5308-5316.

13. Rabinowitz JD, White E. Autophagy and metabolism. Science. 2010; 330(6009):1344-1348.

14. Giat E, Ehrenfeld M, Shoenfeld Y. Cancer and autoimmune diseases. Autoimmun Rev. 2017;16(10):1049-1057.

15. Bureau of National Health Insurance. National Health Insurance Annual Statistical Report, 2016-2017 (bilingual). Available from: https://www. nhi.gov.tw/Resource/webdata/13767_1_National\%20Health\%20Insurance\%20in\%20Taiwan\%202016-2017(bilingual).pdf

16. Charlson ME, Pompei P, Ales KL, Mackenzie CR. A new method of classifying prognostic comorbidity in longitudinal studies: development and validation. J Chronic Dis. 1987;40(5):373-383.

17. Melles RB, Marmor MF. The risk of toxic retinopathy in patients on long-term hydroxychloroquine therapy. JAMA Ophthalmol. 2014; 132(12):1453-1460.

18. Shi TT, Yu XX, Yan LJ, Xiao HT. Research progress of hydroxychloroquine and autophagy inhibitors on cancer. Cancer Chemother Pharmacol. 2017;79(2):287-294.

19. Fang Y, Chen Y, Chung T, et al. Hydroxychloroquine and risk of cancer in patients with primary Sjögren syndrome: propensity score matched landmark analysis. Oncotarget. 2017;8(46):80461-80471.

20. Mathew R, Karp CM, Beaudoin B, et al. Autophagy suppresses tumorigenesis through elimination of p62. Cell. 2009;137(6):1062-1075.

21. Komatsu M, Waguri S, Koike M, et al. Homeostatic levels of p62 control cytoplasmic inclusion body formation in autophagy-deficient mice. Cell. 2007;131(6):1149-1163.

22. Komatsu M, Waguri S, Ueno T, et al. Impairment of starvation-induced and constitutive autophagy in Atg7-deficient mice. J Cell Biol. 2005; 169(3):425-434.

23. White E. Deconvoluting the context-dependent role for autophagy in cancer. Nat Rev Cancer. 2012;12(6):401-410.

24. K-H Y, Kuo C-F, Huang LH, Huang W-K, See L-C. Cancer risk in patients with inflammatory systemic autoimmune rheumatic diseases. Medicine. 2016;95(18):e3540.

25. Goldberg SB, Supko JG, Neal JW, et al. A phase I study of erlotinib and hydroxychloroquine in advanced non-small-cell lung cancer. $J$ Thorac Oncol. 2012;7(10):1602-1608.

\section{Publish your work in this journal}

Therapeutics and Clinical Risk Management is an international, peerreviewed journal of clinical therapeutics and risk management, focusing on concise rapid reporting of clinical studies in all therapeutic areas, outcomes, safety, and programs for the effective, safe, and sustained use of medicines. This journal is indexed on PubMed Central, CAS,

\section{Dovepress}

EMBase, Scopus and the Elsevier Bibliographic databases. The manuscript management system is completely online and includes a very quick and fair peer-review system, which is all easy to use. Visit http://www.dovepress.com/testimonials.php to read real quotes from published authors. 